\title{
Structural characterization of three distinct beta-glucuronidases from the gut commensal bacterium Roseburia hominis
}

\author{
M Gibbs ${ }^{1}$, S Ervin ${ }^{2}$, M Redinbo ${ }^{3}$ \\ ${ }^{1}$ UNC at Chapel Hill, Carrboro, NC, ${ }^{2}$ University of North Carolina at Chapel Hill, Carrboro, NC, ${ }^{3}$ UNC \\ Chapel Hill \\ gibbsm@live.unc.edu
}

Glycoside hydrolase enzymes encoded by the gut microbiome play a key role in the breakdown of a variety of dietary carbohydrates and glycoconjugates. The benefits of this process are two-fold: the human host expands its metabolic potential and gains dietary energy, and the microbiota gain an energy source from the products of this breakdown. Here, we examine the structure and function of three $\beta$-glucuronidase (GUS) glycoside hydrolases from Roseburia hominis, a gut commensal microbe. GUS structures solved using both X-ray crystallography and cryoEM reveal differences in their tertiary and quaternary structures. RhGUS1 contains two unique loops, one that encloses the active site and another with $\alpha$-helical structure. Additionally, RhGUS1 is a tetramer, resulting in a solvent-occluded active site. Conversely, RhGUS2 and RhGUS3 are both dimers with solvent-exposed active sites. These GUSs also contain a C-terminal domain and a FMN-binding site in the beta-sandwich region that are not observed in RhGUS1. These structural differences result in differential processing of several endogenous and exogenous glucuronide substrates in vitro. Together, these data indicate that the structural variation of GUS enzymes from Roseburia hominis allows for this gut microbe to process a range of structurally diverse glucuronide substrates.

Acta Cryst. (2020). A76, a5 